PROCEEDINGS OF THE

AMERICAN MATHEMATICAL SOCIETY

Volume 130, Number 7, Pages 2097-2106

S 0002-9939(01)06280-3

Article electronically published on December 27, 2001

\title{
MARTIN'S AXIOM IS CONSISTENT WITH THE EXISTENCE OF NOWHERE TRIVIAL AUTOMORPHISMS
}

\author{
SAHARON SHELAH AND JURIS STEPRĀNS
}

(Communicated by Alan Dow)

\begin{abstract}
Martin's Axiom does not imply that all automorphisms of $\mathcal{P}(\mathbb{N})$ / $[\mathbb{N}]^{<\aleph_{0}}$ are somewhere trivial. An alternate method for obtaining models where every automorphism of $\mathcal{P}(\mathbb{N}) /[\mathbb{N}]<\aleph_{0}$ is somewhere trivial is explained.
\end{abstract}

\section{INTRODUCTION}

In [5] Veličković constructed a model of Martin's Axiom in which there is a non-trivial automorphism of $\mathcal{P}(\mathbb{N}) /[\mathbb{N}]^{<\aleph_{0}}$. As well as answering a question posed in [4], this put into context another result of [5] showing that the conjunction of MA and OCA implies that all automorphisms are trivial. However, the nontrivial automorphim constructed by Veličković is trivial on many infinite subsets of the integers. Indeed, it was shown in [3] that this is unavoidable since every automorphism of $\mathcal{P}(\mathbb{N}) /[\mathbb{N}]^{<\aleph_{0}}$ is somewhere trivial in Veličković's model of [5].

Hence, the question arises of whether or not Martin's Axiom alone is sufficient to imply that, while there may be non-trivial automorphisms, nevertheless, all automorphisms of $\mathcal{P}(\mathbb{N}) /[\mathbb{N}]^{<\aleph_{0}}$ are somewhere trivial. The main result of this paper is that this is not the case.

The last section presents a simple, alternate method for obtaining models where all automorphisms are somewhere trivial. It has the advantage that it can produce models where $\mathfrak{d}=\aleph_{1}$ whereas the oracle chain condition method adds Cohen reals and so does not achieve this.

\section{MARTIN's AXIOM AND A NOWHERE TRIVIAL AUTOMORPHISM}

If $\alpha$ and $\beta$ are ordinals, then the notation $[\alpha, \beta)$ will be used to denote the set $\beta \backslash \alpha$. The relations $\equiv^{*}, \subseteq^{*}$ and $\supseteq^{*}$ will have the usual meaning as relations on subsets of the integers modulo a finite set. The convention on forcing partial orders will be that larger conditions force more information.

Received by the editors October 12, 2000 and, in revised form, January 12, 2001.

1991 Mathematics Subject Classification. Primary 03E50, 03E35.

Key words and phrases. Boolean algebra, Martin's Axiom, automorphism.

The research of the first author was supported by The Israel Science Foundation founded by the Israel Academy of Sciences and Humanities, and by NSF grant No. NSF-DMS97-04477. Research of the second author for this paper was partially supported by NSERC of Canada. This is paper number 735 in the first author's personal listing. 
Definition 2.1. If $W$ is a set of ordinals, then the indexed family

$$
\mathfrak{S}=\left\{\left(A_{\xi}, F_{\xi}, \mathfrak{B}_{\xi}\right)\right\}_{\xi \in W}
$$

will be said to be a tower of permutations if:

(1) $A_{\xi} \subseteq \mathbb{N}$ and $F_{\xi}$ is a permutation of $\mathbb{N}$ for each $\xi$,

(2) $F_{\xi}\left\lceil m\right.$ is a permutation of $m$ for each $m$ in $A_{\xi}$,

(3) $\mathfrak{B}_{\xi}$ is a finite subalgebra of $\mathcal{P}(\mathbb{N})$ for each $\xi$,

(4) if $\xi \in \zeta$, then $\mathfrak{B}_{\xi} \subseteq \mathfrak{B}_{\zeta}$ and $A_{\xi} \supseteq^{*} A_{\zeta}$,

(5) if $\xi \in \zeta$, then $F_{\zeta}(B) \equiv{ }^{*} F_{\xi}(B)$ for each $B \in \mathfrak{B}_{\xi}$.

Define $\mathbb{Q}(\mathfrak{S})$ to be the set consisting of all quadruples $p=\left(a^{p}, f^{p}, \alpha^{p}, \mathfrak{B}^{p}\right)$ such that:

(1) $a^{p} \subseteq \mathbb{N}$ is a finite subset,

(2) $f^{p}\left\lceil m\right.$ is a permutation of $m$ for each $m \in a^{p}$ and the domain of $f^{p}$ is $\max \left(a^{p}\right)$

(3) $\alpha^{p} \in W$,

(4) $\max \left(a^{p}\right) \in A_{\alpha^{p}}$,

and the relation $\leq$ on $\mathbb{Q}(\mathfrak{S})$ is defined by $p \leq q$ if and only if

$$
\begin{gathered}
a^{p} \subseteq a^{q}, \quad\left(\max \left(a^{p}\right)+1\right) \cap a^{q}=a^{p}, \quad f^{p} \subseteq f^{q}, \quad \mathfrak{B}^{p} \subseteq \mathfrak{B}^{q}, \quad \alpha^{p} \subseteq \alpha^{q}, \\
\left(A_{\alpha^{q}} \backslash \max \left(a^{q}\right)\right) \cup\left(a^{q} \backslash a^{p}\right) \subseteq A_{\alpha^{p}},
\end{gathered}
$$

and, for each $B$ belonging to $\mathfrak{B}^{p} \cap \mathfrak{B}_{\alpha^{p}}$, the following two conditions hold:

$$
\begin{aligned}
& \left(\forall\{n, m\} \in\left[a^{q} \backslash \max \left(a^{p}\right)\right]^{2}\right) f^{q}(B \cap[m, n))=F_{\alpha^{p}}(B \cap[m, n)), \\
& \left(\forall\{n, m\} \in\left[A_{\alpha^{q}} \backslash \max \left(a^{q}\right)\right]^{2}\right) F_{\alpha^{q}}(B \cap[m, n))=F_{\alpha^{p}}(B \cap[m, n)) .
\end{aligned}
$$

If $G$ is generic for $\mathbb{Q}(\mathfrak{S})$, then define $A_{\mathfrak{S}}[G]=\bigcup_{p \in G} a^{p}$ and $F_{\mathfrak{S}}[G]=\bigcup_{p \in G} f^{p}$.

Lemma 2.1. For any tower of permutations $\mathfrak{S}$ the structure $(\mathbb{Q}(\mathfrak{S}), \leq)$ is a partial order.

Proof. That $(\mathbb{Q}(\mathfrak{S}), \leq)$ is reflexive and antisymmetric is obvious. To prove transitivity suppose that $p \leq q$ and $q \leq r$. Condition (2.1) for $p \leq r$ is easily seen to be satisfied. To see that condition (2.2) for $p \leq r$ is satisfied note that

$$
A_{\alpha^{r}} \backslash \max \left(a^{r}\right) \subseteq A_{\alpha^{q}} \backslash \max \left(a^{r}\right) \subseteq A_{\alpha^{q}} \backslash \max \left(a^{q}\right) \subseteq A_{\alpha_{p}}
$$

and that

$$
a^{r} \backslash a^{p} \subseteq\left(a^{r} \backslash a^{q}\right) \cup\left(a^{q} \backslash a^{p}\right) \subseteq A_{\alpha^{q}} \backslash \max \left(a^{q}\right) \cup A_{\alpha^{p}} \subseteq A_{\alpha^{p}}
$$

which shows that $\left(A_{\alpha^{r}} \backslash \max \left(a^{r}\right)\right) \cup\left(a^{r} \backslash a^{p}\right) \subseteq A_{\alpha^{p}}$, as required.

To show that conditions (2.3) and (2.4) hold, let $B \in \mathfrak{B}^{p} \cap \mathfrak{B}_{\alpha^{p}}$. Given any pair $\{n, m\} \in\left[a^{r} \backslash \max \left(a^{p}\right)\right]^{2}$, it may, without loss of generality, be assumed that $n$ and $m$ are successive elements of $a^{r} \backslash \max \left(a^{p}\right)$. Hence, either $\{n, m\} \in\left[\left(a^{r} \backslash \max \left(a^{q}\right)\right]^{2}\right.$ or $\{n, m\} \in\left[\left(a^{q} \backslash \max \left(a^{p}\right)\right]^{2}\right.$. In the second case, from the fact that $p \leq q$ it immediately follows that $f^{q}(B \cap[n, m))=F_{\alpha^{p}}(B \cap[n, m))$. Since $q \leq r$ it follows that $f^{q} \subseteq f^{r}$ and so $f^{r}(B \cap[n, m))=F_{\alpha^{p}}(B \cap[n, m))$. On the other hand, in the first case $f^{r}(B \cap[n, m))=F_{\alpha^{q}}(B \cap[n, m))$ since $q \leq r$ and $\mathfrak{B}_{\alpha^{p}} \subseteq \mathfrak{B}_{\alpha^{q}}$. Moreover, using condition (2.4) and $p \leq q$ it is possible to conclude that $F_{\alpha^{q}}(B \cap[n, m))=$ $F_{\alpha^{p}}(B \cap[n, m))$. Hence, in either case $f^{r}(B \cap[n, m))=F_{\alpha^{p}}(B \cap[n, m))$ which establishes that condition (2.3) holds for $p \leq r$. 
To see that condition (2.4) holds for $p \leq r$ let $\{n, m\} \in\left[A_{\alpha^{r}} \backslash \max \left(a^{r}\right)\right]^{2}$. It follows from $p \leq q$ and $q \leq r$ that $F_{\alpha^{q}}(B \cap[n, m))=F_{\alpha^{r}}(B \cap[n, m))$ and, since $\{n, m\} \subseteq A_{\alpha^{r}} \backslash \max \left(a^{r}\right) \subseteq A_{\alpha^{q}} \backslash \max \left(a^{q}\right)$, that $F_{\alpha^{q}}(B \cap[n, m))=F_{\alpha^{p}}(B \cap[n, m))$. Hence, $F_{\alpha^{r}}(B \cap[n, m))=F_{\alpha^{p}}(B \cap[n, m))$, establishing condition (2.4).

Lemma 2.2. Given a tower of permutations $\mathfrak{S}=\left\{\left(A_{\xi}, F_{\xi}, \mathfrak{B}_{\xi}\right)\right\}_{\xi \in W}$, an integer $n, B \in \bigcup_{\xi \in W} \mathfrak{B}_{\xi}$ and $\zeta \in W$ the following sets are dense in $\mathbb{Q}(\mathfrak{S})$ :

$$
\begin{gathered}
\left\{p \in \mathbb{Q}(\mathfrak{S}): \max \left(a^{p}\right)>n\right\}, \\
\left\{p \in \mathbb{Q}(\mathfrak{S}): \alpha^{p} \geq \zeta\right\}, \\
\left\{p \in \mathbb{Q}(\mathfrak{S}): B \in \mathfrak{B}^{p}\right\} .
\end{gathered}
$$

Proof. To prove that the set 2.5 is dense let $p \in \mathbb{Q}(\mathfrak{S})$ and $n$ be given. Let $k \in A^{\alpha^{p}}$ be such that $k>n$. Using condition (4) of Definition 2.1 it follows that $\max \left(a^{p}\right) \in A_{\alpha^{p}}$ and, hence, $F_{\alpha_{p}}\left\lceil\left[\max \left(a^{p}\right), k\right)\right.$ is a permutation of $\left[\max \left(a^{p}\right), k\right)$. Letting $q=\left(a^{p} \cup\{k\}, f^{p} \cup F_{\alpha_{p}}\left\lceil\left[\max \left(a^{p}\right), k\right), \alpha^{p}, \mathfrak{B}^{p}\right)\right.$ it follows that $q \geq p$. Observe for later reference that it has actually been shown that

$$
(\forall p \in \mathbb{Q}(\mathfrak{S}))(\forall n \in \mathbb{N})(\exists q \geq p) \max \left(a^{q}\right)>n \text { and } \alpha^{q}=\alpha^{p} \text { and } \mathfrak{B}^{p}=\mathfrak{B}^{q} .
$$

To prove that the set (2.6) is dense let $p \in \mathbb{Q}(\mathfrak{S})$ and $\zeta \in W$ be given. Since it may as well be assumed that $\zeta>\alpha^{p}$, it is possible to find $n$ so large that $A_{\zeta} \backslash n \subseteq A_{\alpha^{p}}$ and for all $\{i, j\} \in\left[A_{\zeta} \backslash n\right]^{2}$ and $B \in \mathfrak{B}^{p}$

$$
F_{\zeta}(B \cap[i, j))=F_{\alpha^{p}}(B \cap[i, j)) .
$$

Using the set (2.5) of Lemma 2.2 choose $q$ such that $p \leq q$ and $n<\max \left(a^{q}\right)$. From (2.8) it can be assumed that $\mathfrak{B}^{q}=\mathfrak{B}^{p}$ and that $\alpha^{q}=\alpha^{p}$. Now, let $r=$ $\left(a^{q}, f^{q}, \zeta, \mathfrak{B}^{p}\right)$. That conditions (2.2) and (2.4) for the relation $q \leq r$ are satisfied follows from the choice of $n$ while condition (2.1) is obvious. Condition (2.3) has no content in the case of $q \leq r$ since $a^{q}=a^{r}$. Now use transitivity and the fact that $p \leq q$. Observe for later reference that it has actually been shown that

$$
(\forall p \in \mathbb{Q}(\mathfrak{S}))\left(\forall \zeta>\alpha^{p}\right)(\exists q \geq p) \alpha^{q}=\zeta .
$$

There is no problem in proving that the set 2.7) is dense.

Lemma 2.3. If $\mathfrak{S}=\left\{\left(A_{\xi}, F_{\xi}, \mathfrak{B}_{\xi}\right)\right\}_{\xi \in W}$ is a tower of permutations and $p \in \mathbb{Q}(\mathfrak{S})$, then

$$
\begin{aligned}
& p \Vdash_{\mathbb{Q}(\mathfrak{S})} " A_{\mathfrak{S}}[G] \backslash \max \left(a^{p}\right) \subseteq A_{\alpha^{p}} ", \\
& p \Vdash_{\mathbb{Q}(\mathfrak{S})} "\left(\forall B \in \mathfrak{B}^{p} \cap \mathfrak{B}_{\alpha^{p}}\right)\left(\forall\{n, m\} \in\left[A_{\alpha^{p}} \backslash \max \left(a^{p}\right)\right]^{2}\right) \\
& F_{\mathfrak{S}}[G](B \cap[n, m))=F_{\alpha^{p}}(B \cap[n, m)) .
\end{aligned}
$$

Proof. This is standard using condition (2.2) for (2.10) and condition (2.3) for (2.11).

Let $\kappa$ be a regular uncountable cardinal and let $C \subseteq \kappa$ be any set containing 0 and closed under limits of increasing $\omega_{1}$-sequences such that $\kappa \backslash C$ is also unbounded. Now define $\mathbb{P}_{\eta}$, as well as a $\mathbb{P}_{\eta}$-name for a tower of permutations $\mathfrak{S}_{\eta}=\left\{\left(A_{\zeta}, F_{\zeta}, \mathfrak{B}_{\zeta}\right)\right\}_{\zeta \in \eta \cap C}$, by induction on $\eta$. Let $A_{0} \in[\mathbb{N}]^{\aleph_{0}}$ and $F_{0}$ be arbitrary subject to the fact that $F_{0}$ is a permutation of $\mathbb{N}$ such that $F_{0} \uparrow[n, m)$ is a permutation for each $\{n, m\} \subseteq A_{0}$. Let $\mathfrak{B}_{0}=\mathcal{P}(\mathbb{N})$ in the sense of the ground 
model. Then let $\mathfrak{S}_{1}=\left\{\left(A_{0}, F_{0}, \mathfrak{B}_{0}\right)\right\}$ and let $\mathbb{P}_{1}=\mathbb{Q}_{0}$ be Cohen forcing. If $\eta$ is a limit, then $\mathbb{P}_{\eta}$ is simply the finite support limit of $\left\{\mathbb{P}_{\zeta}\right\}_{\zeta \in \eta}$ and $\mathfrak{S}_{\eta}=\bigcup_{\xi \in C \cap \eta} \mathfrak{S}_{\xi}$. If $\eta \notin C$, then $\mathbb{P}_{\eta+1}=\mathbb{P}_{\eta} * \mathbb{Q}_{\eta}$ where $\mathbb{Q}_{\eta}$ is a ccc partial order chosen according to some bookkeeping scheme which will guarantee that Martin's Axiom holds at stage $\kappa$. In this case $\mathfrak{S}_{\eta+1}=\mathfrak{S}_{\eta}$. If $\eta \in C \backslash\{0\}$, then $\mathbb{P}_{\eta+1}=\mathbb{P}_{\eta} * \mathbb{Q}\left(\mathfrak{S}_{\eta}\right)$ and $A_{\eta}$ is defined to be $A_{\mathfrak{S}_{\eta}}[G], F_{\eta}$ is defined to be $F_{\mathfrak{S}_{\eta}}[G]$ where $G$ is the canonical name for the generic set on $\mathbb{Q}\left(\mathfrak{S}_{\eta}\right)$. In this case $\mathfrak{S}_{\eta+1}=\mathfrak{S}_{\eta} \cup\left\{\left(A_{\eta}, F_{\eta}, \mathcal{P}(\mathbb{N}) \cap V^{\mathbb{P}_{\eta}}\right)\right\}$. As usual, if $p \in \mathbb{P}_{\eta}$, then $p\left\lceil\alpha \Vdash_{\mathbb{P}_{\alpha}} " p(\alpha) \in \mathbb{Q}_{\alpha}\right.$ ".

Definition 2.2. Let $\mathbb{P}_{\alpha}^{w} \subseteq \mathbb{P}_{\alpha}$ consist of all those $p \in \mathbb{P}_{\alpha}$ such that there are $k \in \mathbb{N}$, $\Gamma \in[C \cap \alpha]^{<\aleph_{0}}$ and $\left\{\left(a_{\gamma}, f_{\gamma}\right)\right\}_{\gamma \in \Gamma}$ such that:

- $\Gamma=\operatorname{domain}(p) \cap C$,

- $0 \in \Gamma$,

- if $\gamma \in \Gamma$, then $p\left\lceil\gamma \mathbb{P}_{\mathbb{P}_{\gamma}}\right.$ " $p(\gamma)=\left(\check{a}_{\gamma}, \check{f}_{\gamma}, \check{\alpha}_{\gamma}, \mathfrak{B}^{\gamma}\right)$ " for some $\mathfrak{B}^{\gamma}$,

- $\alpha_{\gamma} \in \Gamma \cap \gamma$ for each $\gamma \in \Gamma$,

- if $\gamma \in \Gamma$, then $\max \left(a_{\gamma}\right)=k$,

- if $\gamma$ and $\gamma^{\prime}$ are in $\Gamma$ and $\gamma^{\prime}<\gamma$, then $p\left\lceil\gamma{\Vdash_{\mathbb{P}_{\gamma}}}\right.$ ' $\mathfrak{B}^{\gamma^{\prime}}=\mathfrak{B}^{\gamma} \cap \mathfrak{B}_{\gamma^{\prime}}$ ".

The pair $\left(k,\left\{\left(a_{\gamma}, f_{\gamma}\right)\right\}_{\gamma \in \Gamma}\right)$ will be said to witness that $p \in \mathbb{P}_{\alpha}^{w}$. Let $\mathbb{P}_{\alpha}^{*} \subseteq \mathbb{P}_{\alpha}$ consist of all those $p \in \mathbb{P}_{\alpha}^{w}$ such that, in addition to the other requirements, $\alpha_{\gamma}=\max (\Gamma \cap \gamma)$ for each $\gamma \in \Gamma$.

Lemma 2.4. If $p \in \mathbb{P}_{\alpha}^{w}$ and this is witnessed by $\left(k,\left\{\left(a_{\gamma}, f_{\gamma}\right)\right\}_{\gamma \in \Gamma}\right)$, then:

- if $\gamma \in \Gamma$, then $p\left\lceil\gamma+1 \Vdash_{\mathbb{P}_{\gamma+1}}\right.$ " $A_{\alpha_{\gamma}} \supseteq\left(A_{\gamma} \backslash k\right)$ ",

- if $\gamma \in \Gamma$, then $p\left\lceil\gamma+1 \Vdash_{\mathbb{P}_{\gamma+1}} "\left(\forall B \in \mathfrak{B}^{\gamma} \cap \mathfrak{B}_{\alpha_{\gamma}}\right)\left(\forall\{n, m\} \in\left[A_{\gamma} \backslash k\right]^{2}\right)\right.$ $F_{\gamma}(B \cap[n, m))=F_{\alpha_{\gamma}}(B \cap[n, m)) "$.

Proof. This is an immediate consequence of Lemma 2.3.

Definition 2.3. If $p \in \mathbb{P}_{\alpha}^{w}$ is witnessed by $\left(k,\left\{\left(a_{\gamma}, f_{\gamma}\right)\right\}_{\gamma \in \Gamma}\right)$, then define $p^{+} \in \mathbb{P}_{\alpha}^{*}$ by

$$
p^{+}(\xi)= \begin{cases}p(\xi) & \text { if } \xi \notin \Gamma, \\ \left(a_{\xi}, f_{\xi}, \max (\Gamma \cap \xi), \mathfrak{B}^{\xi}\right) & \text { if } \xi \in \Gamma .\end{cases}
$$

Lemma 2.5. If $p \in \mathbb{P}_{\alpha}^{w}$, then $p^{+} \geq p$.

Proof. Proceed by induction on $\beta \in \alpha$ to show that $p^{+} \uparrow \beta \geq p \uparrow \beta$. Note that the cases $\beta=0$ or $\beta$ a limit pose no problem. Given that $p^{+} \uparrow \beta \geq p\lceil\beta$ let $\Gamma \cap \beta$ be enumerated, in order, by $\left\{\gamma_{1}, \gamma_{2}, \ldots, \gamma_{n}\right\}$. If follows directly from Lemma 2.4 and the definition of $p^{+}$that:

- $p^{+}\left\lceil\beta \Vdash_{\mathbb{P}_{\beta}}\right.$ " $A_{\gamma_{1}} \backslash \check{k} \supseteq\left(A_{\gamma_{2}} \backslash \check{k}\right) \ldots \supseteq\left(A_{\gamma_{n}} \backslash \check{k}\right)$ ",

- $p^{+} \mid \beta \Vdash_{\mathbb{P}_{\beta}} "\left(\forall B \in \mathfrak{B}^{\gamma_{j}} \cap \mathfrak{B}_{\gamma_{i}}\right)\left(\forall\left\{m, m^{\prime}\right\} \in A_{\gamma_{j}} \backslash \check{k}\right) F_{\gamma_{j}}\left(B \cap\left[m, m^{\prime}\right)\right)=$ $F_{\gamma_{i}}\left(B \cap\left[m, m^{\prime}\right)\right)$ " if $i \leq j$.

In particular, noting that there is some $i$ such that $\alpha_{\beta}=\gamma_{i}$,

$$
p^{+}\left\lceil\beta \Vdash_{\mathbb{P}_{\beta}} \text { " } A_{\alpha_{\beta}} \backslash \check{k} \supseteq A_{\gamma_{n}} "\right.
$$

and

$$
\begin{array}{r}
p^{+}\left\lceil\beta \Vdash _ { \mathbb { P } _ { \beta } } " ( \forall B \in \mathfrak { B } ^ { \alpha _ { \beta } } \cap \mathfrak { B } _ { \gamma _ { n } } ) \left(\forall\left\{m, m^{\prime}\right\} \in A_{\left.\alpha_{\beta} \backslash k\right)}\right.\right. \\
F_{\alpha_{\beta}}\left(B \cap\left[m, m^{\prime}\right)\right)=F_{\gamma_{n}}\left(B \cap\left[m, m^{\prime}\right)\right) " .
\end{array}
$$

Hence, $p^{+} \uparrow \beta+1 \geq p \uparrow \beta+1$. 
Lemma 2.6. For each $\alpha \leq \kappa$ the subset $\mathbb{P}_{\alpha}^{w}$ is dense in $\mathbb{P}_{\alpha}$.

Proof. Proceed by induction on $\alpha$ noting that the cases $\alpha \leq 1$ and $\alpha$ a limit are trivial. Therefore, suppose that Lemma 2.6 has been established for $\beta$ and that $p \in \mathbb{P}_{\beta+1}$. Without loss of generality it may be assumed that $\beta \in C$. Choose $p^{\prime} \geq p \uparrow \beta$ and $(a, f, \zeta)$ such that $\zeta \in \beta$ and $p^{\prime} \Vdash_{\mathbb{P}_{\beta}} " p(\beta)=(\check{a}, \check{f}, \check{\zeta}, \mathfrak{B}) "$ and, moreover, $p^{\prime} \Vdash_{\mathbb{P}_{\beta}}$ " $\mathfrak{B}=\left\{B_{i}\right\}_{i \in m}$ " and, for each $i \in m$ the condition $p^{\prime}$ decides the value of the least ordinal $\zeta(i) \in \beta$ such that $B_{i} \in \mathfrak{B}_{\zeta(i)}$. Furthermore, it may, without loss of generality, be assumed that $\{\zeta\} \cup\{\zeta(i)\}_{i \in m} \subseteq \operatorname{domain}\left(p^{\prime}\right)$ and that

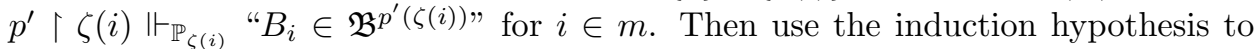
find $p^{\prime \prime} \in \mathbb{P}_{\beta}^{w}$ extending $p^{\prime}$ and such that $\left(k,\left\{\left(a_{\gamma}, f_{\gamma}\right)\right\}_{\gamma \in \Gamma}\right)$ witnesses that $p^{\prime \prime} \in \mathbb{P}_{\beta}^{w}$. Without loss of generality, $k \geq \max (a)$. Now, the fact that $\{\max (a), k\} \subseteq A_{\zeta}$ implies that $F_{\zeta} \uparrow[\max (a), k)$ is a permutation of $[\max (a), k)$. Hence it is possible to define $f^{\prime}=f \cup F_{\zeta} \uparrow[\max (a), k)$ and $a^{\prime}=a \cup\{k\}$. Then define $q$ so that

$$
q(\xi)= \begin{cases}p^{\prime \prime}(\xi) & \text { if } \xi \in \beta, \\ \left(a^{\prime}, f^{\prime}, \zeta, \mathfrak{B} \cup \bigcup_{\gamma \in \Gamma} \mathfrak{B}^{p^{\prime}(\gamma)}\right) & \text { if } \xi=\beta,\end{cases}
$$

and note that $q \in \mathbb{P}_{\beta+1}^{w}$ and $q \geq p$.

Corollary 2.1. For each $\alpha \leq \kappa$ the subset $\mathbb{P}_{\alpha}^{*}$ is dense in $\mathbb{P}_{\alpha}$.

Proof. This is immediate from Lemma 2.6 and Lemma 2.5 .

Lemma 2.7. Given that $p \in \mathbb{P}_{\alpha}^{w}$ and that this is witnessed by $\left(k,\left\{\left(a_{\gamma}, f_{\gamma}\right)\right\}_{\gamma \in \Gamma}\right)$ and $\mu \in(C \cap \alpha) \backslash$ domain $(p)$ then the following condition $p\langle\mu\rangle$ extends $p$ and is also in $\mathbb{P}_{\alpha}^{w}$ :

$$
p\langle\mu\rangle(\xi) \begin{cases}p(\xi) & \text { if } \xi \neq \mu, \\ \left(\{k\},\{(j, j)\}_{j \in k}, 0, \bigcup_{\gamma \in \Gamma \cap \mu} \mathfrak{B}^{p(\gamma)}\right) & \text { if } \xi=\mu .\end{cases}
$$

Proof. Notice that since $\mu \notin \operatorname{domain}(p)$ the restrictions on extension do not apply and it is easy to check that the condition $\left(\{k\},\{(j, j)\}_{j \in k}, \max (\Gamma \cap \mu), \bigcup_{\gamma \in \Gamma \cap \mu} \mathfrak{B}^{p(\gamma)}\right)$ belongs to $\mathbb{Q}_{\mu}$. Since $p \in \mathbb{P}_{\alpha}^{w}$ it follows that $k \in A_{0}$ and, hence, that $p\langle\mu\rangle \in \mathbb{P}_{\alpha}^{w}$. That $p\langle\mu\rangle \geq p$ is immediate from the definition.

Lemma 2.8. Suppose that $p$ and $q$ are conditions in $\mathbb{P}_{\alpha}$ such that:

- $p \in \mathbb{P}_{\alpha}^{*}$ is witnessed by $\left(k,\left\{\left(a_{p}^{\gamma}, f_{p}^{\gamma}\right)\right\}_{\gamma \in \Gamma_{p}}\right)$,

- $q \in \mathbb{P}_{\alpha}^{*}$ is witnessed by $\left(k,\left\{\left(a_{q}^{\gamma}, f_{q}^{\gamma}\right)\right\}_{\gamma \in \Gamma_{q}}\right)$,

- $\max (\operatorname{domain}(p))=\max (\operatorname{domain}(q))=\bar{\gamma} \in \Gamma_{p} \cap \Gamma_{q}$,

- $\left(a_{q}^{\bar{\gamma}}, f_{q}^{\bar{\gamma}}\right)=\left(a_{p}^{\bar{\gamma}}, f_{p}^{\bar{\gamma}}\right)$,

- $\max (\operatorname{domain}(q)) \cap \bar{\gamma}<\min (\operatorname{domain}(p) \backslash\{0\})$,

- $p(0)$ and $q(0)$ are compatible.

Under these conditions $p$ and $q$ are compatible.

Proof. Let $\sigma$ be the maximum member of $\Gamma_{q} \backslash\{\bar{\gamma}\}$. Define $q \sqcup p$ by

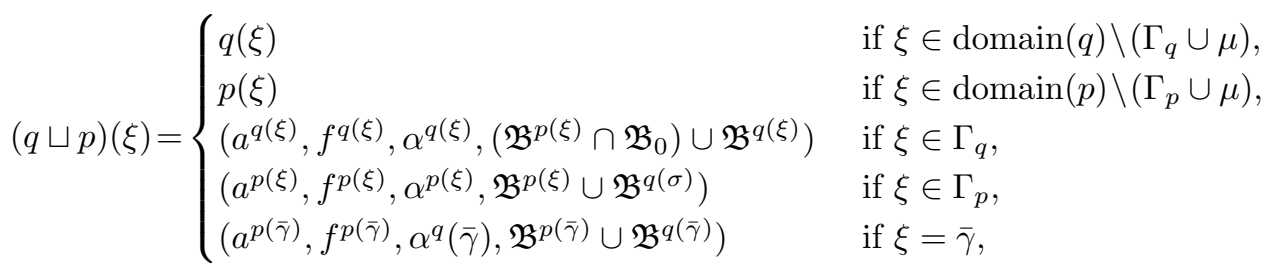


and define $p \sqcup q$ by

$$
(p \sqcup q)(\xi)= \begin{cases}q(\xi) & \text { if } \xi \in \text { domain }(q) \backslash\left(\Gamma_{q} \cup \mu\right), \\ p(\xi) & \text { if } \xi \in \text { domain }(p) \backslash\left(\Gamma_{p} \cup \mu\right), \\ \left(a^{q(\xi)}, f^{q(\xi)}, \alpha^{q(\xi)},\left(\mathfrak{B}^{p(\xi)} \cap \mathfrak{B}_{0}\right) \cup \mathfrak{B}^{q(\xi)}\right) & \text { if } \xi \in \Gamma_{q}, \\ \left(a^{p(\xi)}, f^{p(\xi)}, \alpha^{p(\xi)}, \mathfrak{B}^{p(\xi)} \cup \mathfrak{B}^{q(\sigma)}\right) & \text { if } \xi \in \Gamma_{p}, \\ \left(a^{p(\bar{\gamma})}, f^{p(\bar{\gamma})}, \alpha^{p}(\bar{\gamma}), \mathfrak{B}^{p(\bar{\gamma})} \cup \mathfrak{B}^{q(\bar{\gamma})}\right) & \text { if } \xi=\bar{\gamma} .\end{cases}
$$

(The only difference is to be found in the last lines of the two definitions.) It is easy to check that $p \leq p \sqcup q$ and $q \leq q \sqcup p$ and that both $p \sqcup q$ and $q \sqcup p$ belong to $\mathbb{P}_{\alpha}^{w}$. Moreover $(p \sqcup q)^{+}=(q \sqcup p)^{+}$. Hence $q$ and $p$ are compatible.

Lemma 2.9. If the cofinality of $\zeta \cap C$ is not $\omega_{1}$ and $\mathfrak{S}=\left\{\left(A_{\xi}, F_{\xi}, \mathfrak{B}_{\xi}\right)\right\}_{\xi \in \zeta \cap C}$ is a tower of permutations, then $\mathbb{Q}(\mathfrak{S})$ has property $K$.

Proof. This is standard. If the cofinality of $\zeta \cap C$ is less than $\omega_{1}$, then choose a countable, cofinal subset $C^{\prime}$ of $\zeta \cap C$. Using observation (2.9) of Lemma 2.2 it follows that the set of all $p \in \mathbb{Q}(\mathfrak{S})$ such that $\alpha^{p} \in C^{\prime}$ is dense. It follows that $\mathbb{Q}(\mathfrak{S})$ has a $\sigma$-centred dense subset.

On the other hand, if the cofinality of $\zeta \cap C$ is greater than $\omega_{1}$ and $\left\{p_{\xi}\right\}_{\xi \in \omega_{1}} \subseteq$ $\mathbb{Q}(\mathfrak{S})$ it is possible to choose $\theta \in C \cap \zeta$ such that $\alpha^{p_{\xi}}<\theta$ for each $\xi$. Using observation (2.9) of Lemma 2.2 it may be assumed, by extending each condition, that $\alpha^{p_{\xi}}=\theta$ for each $\xi$. Now there are $a$ and $f$ and an uncountable set of $\xi$ such that $a^{p_{\xi}}=a$ and $f^{p_{\xi}}=f$. Any two of these are easily seen to be compatible.

The fact that the tower of permutations needs to be generic, or at least some other condition must be satisfied, in order for Lemma 2.9 to hold has been observed in Theorem 2 of [2].

Lemma 2.10. $\mathbb{P}_{\alpha}$ has the countable chain condition for each $\alpha$.

Proof. Proceed by induction on $\alpha$. If $\alpha=1$ the result is immediate and if $\alpha$ is a limit the result follows from the induction hypothesis and the finite support of the iteration. Therefore consider the case $\alpha=\beta+1$ and assume that the countable chain condition has already been established for $\mathbb{P}_{\beta}$.

Next, observe that if $C \cap \beta$ is not cofinal in $\beta$, then the induction hypothesis is easily applied since, in this case, $C$ has a maximal element below $\beta$ and so $\mathbb{Q}_{\beta}$ has the countable chain condition by Lemma 2.9. If $C \cap \beta$ has cofinality different from $\omega_{1}$, then once again Lemma 2.9 implies that $\mathbb{Q}_{\beta}$ has the countable chain condition; therefore, in either case, so does $\mathbb{P}_{\beta+1}$. Hence it remains to consider the case that $C \cap \beta$ is cofinal in $\beta$ and has cofinality $\omega_{1}$. From the hypothesis on $C$ and the fact that $\beta$ must be the limit of $C \cap \beta$ it follows that $\beta \in C$. By appealing to Lemma 2.6 and extending the conditions in question, it is possible to guarantee that each $p_{\xi}$ is in $\mathbb{P}_{\alpha}^{*}$ and that this is witnessed by $\left(k_{\xi},\left\{\left(a_{\xi}^{\gamma}, f_{\xi}^{\gamma}\right)\right\}_{\gamma \in \Gamma_{\xi}}\right)$. As well, by thinning out, it may be assumed that there is $k$ such that $k_{\xi}=k$ for each $\xi$, and there is a pair $(a, f)$ such that $\left(a_{\xi}^{\beta}, f_{\xi}^{\beta}\right)=(a, f)$ for each $\xi$ and that $\left\{\operatorname{domain}\left(p_{\xi}\right)\right\}_{\xi \in \omega_{1}}$ form a $\Delta$-system with root $\Delta$. Using the fact that $\beta$ is a limit, choose some $\mu \in \beta \cap C$ such that there is some uncountable $\Lambda \subseteq \omega_{1}$ such that if $\{\eta, \zeta\} \in[\Lambda]^{2}$ and $\eta \in \zeta$, then $\max \left(\operatorname{domain}\left(p_{\eta} \cap \beta\right)\right)<\min \left(\operatorname{domain}\left(p_{\zeta} \cap[\mu, \beta)\right)\right)$. It may as well be assumed that $\mu \notin \operatorname{domain}\left(p_{\eta}\right)$ for each $\eta \in \Lambda$. Then use Lemma 2.7] to extend each $p_{\eta}$ to some $p_{\eta}\langle\mu\rangle^{+}$. 
Now, using the induction hypothesis, find $\{\eta, \zeta\} \in[\Lambda]^{2}$ such that $\eta \in \zeta$ and $p_{\eta}\langle\mu\rangle^{+} \mid \mu+1$ is compatible with $p_{\zeta}\langle\mu\rangle^{+} \mid \mu+1$. Choose $r \in \mathbb{P}_{\mu+1}$ extending both $p_{\eta}\langle\mu\rangle^{+}\left\lceil\mu+1\right.$ and $p_{\zeta}\langle\mu\rangle^{+} \mid \mu+1$.

Let $G \subseteq \mathbb{P}_{\mu+1}$ be generic over $V$ and containing $r$. Observe that $\mathbb{P}_{\alpha} / \mathbb{P}_{\mu+1}$ as interpreted in $V[G]$ is a partial order like $\mathbb{P}_{\alpha}$ in $V$ except that the first factor of $\mathbb{P}_{\alpha} / \mathbb{P}_{\mu+1}$ is $\mathbb{Q}\left(\left\{\left(A_{\mu}, F_{\mu}, \mathcal{P}(\mathbb{N}) \cap V[G]\right)\right\}\right)$. Since $r \Vdash_{\mathbb{P}_{\mu+1}}$ " $k \in A_{\mu}$ " it follows that $k \in A_{\mu}$ in $V[G]$. It follows that $p_{\eta}\langle\mu\rangle^{+} \uparrow[\mu, \alpha)$ and $p_{\zeta}\langle\mu\rangle^{+} \uparrow[\mu, \alpha)$ belong to $\left(\mathbb{P}_{\alpha} / \mathbb{P}_{\mu+1}\right)^{*}$. Now use Lemma 2.8 in $V[G]$ to conclude that $p_{\eta}\langle\mu\rangle^{+} \uparrow[\mu, \alpha)$ and $p_{\zeta}\langle\mu\rangle^{+} \uparrow[\mu, \alpha)$ are compatible. Hence, so are $p_{\eta}$ and $p_{\zeta}$.

Lemma 2.11. Let $\mathfrak{S}=\left\{\left(A_{\xi}, F_{\xi}, \mathfrak{B}_{\xi}\right)\right\}_{\xi \in W}$ be a tower of permutations and suppose that $A \in[\mathbb{N}]^{\aleph_{0}}$ and $\psi$ is a one-to-one function from $A$ to $\mathbb{N}$. Then $\mathbb{Q}(\mathfrak{S})$ forces that there are infinitely many $a \in A$ such that $F_{\mathfrak{S}}[G](a) \neq \psi(a)$.

Proof. Let $p \in \mathbb{Q}(\mathfrak{S})$ and suppose that $p \Vdash_{\mathbb{Q}(\mathfrak{S})} "(\forall a \in \check{A} \backslash \check{k}) \psi(a)=F_{\mathfrak{S}}[G](a)$ " for some integer $k$. From Lemma 2.2 it can be assumed that $k \leq \max \left(a^{p}\right)$. Now choose $m \in A_{\alpha^{p}}$ so large that there exist distinct integers $i$ and $j$ in $\left[\max \left(a^{p}\right), m\right) \cap A$ such that $i \in B$ if and only if $j \in B$ for all $B \in \mathfrak{B}^{p}$. It is possible to choose a bijection $g:\{i, j\} \rightarrow\left\{F_{\alpha^{p}}(i), F_{\alpha^{p}}(j)\right\}$ such that $g \neq \psi \uparrow\{i, j\}$. However, letting

$$
f=F_{\alpha^{p}} \uparrow\left(\left[\max \left(a^{p}\right), m\right) \backslash\{i, j\}\right) \cup g
$$

it follows that $f\left(B \cap\left[\max \left(a^{p}\right), m\right)\right)=F_{\alpha^{p}}\left(B \cap\left[\max \left(a^{p}\right), m\right)\right)$ for each $B \in \mathfrak{B}^{p}$. Hence $p \leq q=\left(a^{p} \cup\{m\}, f^{p} \cup f, \alpha^{p}, \mathfrak{B}^{p}\right)$ and $q \Vdash_{\mathbb{Q}(\mathfrak{S})} " \check{\psi}(\breve{i}) \neq F_{\mathfrak{S}}[G](\check{i}) "$.

Lemma 2.9 and Lemma 2.11 in the case when $C \cap \zeta$ has a maximal element, together imply that there is a trivial automorphism $\Phi$ of $\mathcal{P}(\mathbb{N}) /[\mathbb{N}]^{<\aleph_{0}}$ and a $\sigma$-centred forcing $\mathbb{P}$ such that $\Phi$ can be extended to a trivial automorphism of $\mathcal{P}(\mathbb{N}) /[\mathbb{N}]<\aleph_{0}$ in two different ways in the generic extension by $\mathbb{P}$. Hence, these lemmas can be thought of as strengthening the folklore result that certain automorphisms - such as the identity - can be extended to generic trivial automorphisms by the natural $\sigma$-centred forcing; in other words, the forcing which approximates the generic permutation by finite permutations and promises to respect the identity on certain, finitely many, infinite sets.

Lemma 2.12. Let $\mathfrak{S}=\left\{\left(A_{\xi}, F_{\xi}, \mathfrak{B}_{\xi}\right)\right\}_{\xi \in W}$ be a tower of permutations such that $W$ has a maximal element $\theta$. If $A$ belongs to $[\mathbb{N}]^{\aleph_{0}}$, but not necessarily to $\mathfrak{B}_{\theta}$, and $G$ is $\mathbb{Q}(\mathfrak{S})$ generic over $V$, then there are infinitely many integers $j \in A$ such that $F_{\mathfrak{S}}[G](j)=F_{\theta}(j)$.

Proof. This is a standard use of genericity.

Theorem 2.1. Given any regular, uncountable cardinal $\kappa$, it is consistent relative to the consistency of set theory that Martin's Axiom holds, $2^{\aleph_{0}}=\kappa$ and there is a nowhere trivial automorphism of $\mathcal{P}(\mathbb{N})$ modulo the finite sets.

Proof. Let $C \subseteq \kappa$ be a closed unbounded set such that $\kappa \backslash C$ is unbounded. Construct a finite support iteration $\left\{\mathbb{P}_{\xi}\right\}_{\xi \in \kappa}$ so that Martin's Axiom is forced to hold by the iteration on $\kappa \backslash C$ and such that a tower of permutations $\left\{\left(A_{\xi}, F_{\xi}, \mathfrak{B}_{\xi}\right)\right\}_{\xi \in \kappa}$ is generically constructed along $C$ such that $\mathfrak{B}_{\xi}=\mathcal{P}(\mathbb{N}) \cap V\left[\mathbb{P}_{\xi} \cap G\right]$ where $G$ is $\mathbb{P}_{\kappa}$ generic over $V$. Then let $\Psi$ be the automorphism of $\mathcal{P}(\mathbb{N}) /[\mathbb{N}]^{<\aleph_{0}}$ defined by $\Psi([A])=[B]$ if and only if there is some $\xi \in \kappa$ such that $F_{\xi}(A) \equiv{ }^{*} B$ and $A \in \mathfrak{B}_{\xi}$. To see that $\Psi$ is nowhere trivial suppose that $\Psi$ is induced by $\psi$ on $A$. Let $\xi \in C$ be 
an ordinal large enough that $A$ and $\psi$ both belong to $V\left[G \cap \mathbb{P}_{\xi}\right]$. Using Lemma 2.11 it is possible to find in $V\left[G \cap \mathbb{P}_{\xi+1}\right]$ an infinite set $Z$ such that $\psi(Z) \cap F_{\xi}(Z)=\emptyset$. However, there is no guarantee that $Z$ belongs to $\mathfrak{B}_{\xi}$. Let $\rho$ be the first member of $C$ greater than $\xi$. Then $Z$ does belong to $\mathfrak{B}_{\rho}$. From Lemma 2.12 it follows that $F_{\rho}(j)=F_{\xi}(j)$ for infinitely many $j$ belonging to $Z$. Therefore $F_{\rho}(Z) \backslash \psi(Z)$ is infinite contradicting that $\psi$ induces $\Psi$ on $A$ and $\left[F_{\rho}(Z)\right]=\Psi([Z])$.

It should be noted that Theorem 2.1 would be of interest even if Martin's Axiom did not hold in the model constructed since it would still provide a method for constructing models of set theory with nowhere trivial automorphisms of $\mathcal{P}(\mathbb{N}) /[\mathbb{N}]^{<\aleph_{0}}$ and the continuum arbitrarily large.

\section{Ruining automorphisms with Silver reals}

Definition 3.1. Suppose that $\mathbb{Q}$ and $\mathbb{P}$ are partial orders such that $\mathbb{Q}$ is completely embedded in $\mathbb{P}$ and that $\mathbb{Q}$ is Suslin (see [1]). Then $\mathbb{P}$ will be said to be sufficiently Suslin over $\mathbb{Q}$ if for every $\mathbb{P}$-name $g$ for a function from $\omega$ to 2 there is a dense set of $p \in \mathbb{P}$ such that

$$
\left\{(q, f) \in \mathbb{Q} \times 2^{\omega} \mid\left(\exists p^{\prime} \in \mathbb{P}\right) p^{\prime} \geq q \text { and } p^{\prime} \geq p \text { and } p^{\prime} \Vdash_{\mathbb{P}} " g=\check{f} "\right\}
$$

is analytic.

Lemma 3.1. Any countable support iteration of a combination of Silver reals and Sacks reals is sufficiently Suslin over its first coordinate.

Proof. Let the iteration be obtained from the sequence $\left\{\mathbb{P}_{\xi}\right\}_{\xi \in \eta}$ where each successor stage is constructed by using one of the mentioned partial orders and let the $\mathbb{P}_{\eta}$-name $g$ be given. Let $\mathfrak{M} \prec\left(H\left(\left|\mathbb{P}_{\eta}\right|^{+}\right), \in\right)$ be a countable elementary submodel containing $\mathbb{P}_{\eta}$ and $g$. Let $\mathfrak{M} \cap \eta=\Gamma$, let $\left\{\gamma_{i}\right\}_{i \in \omega}$ enumerate $\Gamma$ and let $\Gamma_{j}=\left\{\gamma_{i}\right\}_{i \in j}$. Standard fusion arguments will allow the construction of a family $\left\{p_{\tau}\right\}_{\tau: \Gamma_{n} \rightarrow 2<n}$ such that:

(1) If $\tau(\gamma) \subseteq \tau^{\prime}(\gamma)$ for each $\gamma$, then $p_{\tau} \leq p_{\tau^{\prime}}$.

(2) If $\tau: \Gamma_{n} \rightarrow 2^{<n}$, then $p_{\tau}$ decides the value of $g(n)$.

(3) If $p(\gamma)$ is defined to be

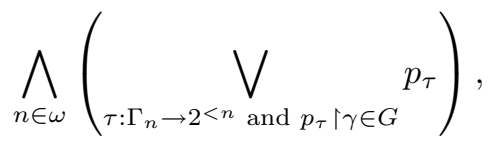

then $p \in \mathbb{P}$.

It follows that for each $q \in \mathbb{P}_{0}$ there is some $p^{\prime} \geq p$ such that $p^{\prime}(0) \geq q$ and $p^{\prime} \Vdash_{\mathbb{P}} " g=\check{f}$ " if and only if, letting $S(f, n)=\left\{\tau \mid \tau: \Gamma_{n} \rightarrow 2^{<n}\right.$ and $p_{\tau} \Vdash_{\mathbb{P}} " g(n)=$ $\check{f}(\check{n})$ " and $\left.p_{\tau}(0) \geq q\right\}$, the set of conditions $\left\{\bigvee_{\tau \in S(f, n)} p_{\tau}\right\}_{n \in \omega}$ has a proper lower bound. For Silver and Sacks forcings checking for a lower bound is easily seen to be $\Sigma_{1}^{1}$ in the parameter defining the fusion sequence; in fact, it is Borel.

Lemma 3.2. If $\mathbb{P}$ is as in Lemma 3.1 and $\Psi$ is an automorphism of $\mathcal{P}(\mathbb{N}) /[\mathbb{N}]<\aleph_{0}$ which is not trivial on any member of $[\mathbb{N}]^{\aleph_{0}}$, then it is not possible to extend $\Psi$ to an automorphism of $\mathcal{P}(\mathbb{N}) /[\mathbb{N}]^{<\aleph_{0}}$ in any generic extension by $\mathbb{S} * \mathbb{P}$.

Proof. Assuming the lemma is false, it is possible to find a condition $(s, p) \in \mathbb{S} * \mathbb{P}$ and an $\mathbb{S} * \mathbb{P}$-name for a set of integers $Z$ such that

$$
(s, p) \Vdash_{\mathbb{S} * \mathbb{P}} \text { " } \Psi^{*} \text { is a lifting of an extension of } \Psi \text { and } \Psi^{*}\left(X_{G}\right)=Z \text { " }
$$


where $X_{G}$ is a name for the generic subset of $\mathbb{N}$ added by $\mathbb{S}$. Let $g=\chi_{Z}$ be the characteristic function of $Z$. Using the fact that $\mathbb{S} * \mathbb{P}$ is sufficiently Suslin, find $\left(s^{\prime}, p^{\prime}\right) \in \mathbb{S} * \mathbb{P}$ such that $\left(s^{\prime}, p^{\prime}\right) \geq(s, p)$ and

$$
\left\{(\bar{s}, f) \in \mathbb{S} \times 2^{\omega} \mid(\exists q \in \mathbb{S} * \mathbb{P}) q(0) \supseteq \bar{s} \text { and } q \geq\left(s^{\prime}, p^{\prime}\right) \text { and } q \Vdash_{\mathbb{S} * \mathbb{P}} " g=\check{f} "\right\}
$$

is analytic. Let $A$ be an infinite set of integers disjoint from the domain of $s^{\prime}$ such that domain $\left(s^{\prime}\right) \cup A$ has infinite complement in the integers. It follows that if $R$ is defined to be $\left\{(t, f) \in 2^{A} \times 2^{\omega} \mid\left(\exists q \geq\left(t \cup s^{\prime}, p^{\prime}\right)\right) q \Vdash_{\mathbb{S *} \mathbb{P}}\right.$ " $g=\check{f}$ " $\}$, then $R$ is analytic.

There are two possibilities. First, suppose that the domain of $R$ is all of $2^{A}$. In this case it is possible to find a continuous function $S$ defined on a comeagre subset of $\mathcal{P}(A)$ such that $R\left(t, S\left(t^{-1}\{1\}\right)\right)$ holds for all $t$ in the domain of $R$. Now let $\psi$ : domain $(S) \rightarrow \mathcal{P}\left(\Psi^{*}(A)\right)$ be defined by

$$
\psi(W)=\left\{n \in \Psi^{*}(A) \mid S(W)(n)=1\right\}
$$

and observe that $\psi$ is continuous. Furthermore, $\psi(W) \equiv{ }^{*} \Psi^{*}(W)$. To see this, let $q \in \mathbb{S} * \mathbb{P}$ be any condition witnessing that $R\left(\chi_{W}\lceil A, S(W))\right.$ holds. Observe that not only does $q$ force $X_{G} \cap A=W$ but also $q \Vdash_{\mathbb{S} * \mathbb{P}}$ " $Z \cap \Psi^{*}(A)=\psi(W)$ ". Hence, $q \Vdash_{\mathbb{S} * \mathbb{P}} " \psi(W)=\Psi^{*}\left(X_{G}\right) \cap \Psi^{*}(A) \equiv{ }^{*} \Psi^{*}\left(X_{G} \cap A\right) \equiv{ }^{*} \Psi^{*}(W)$ ". Therefore, since $\psi(W)$ and $\Psi^{*}(W)$ are sets in the ground model, it follows from the absoluteness of $\equiv^{*}$ that $\psi(W) \equiv{ }^{*} \Psi^{*}(W)$. But now it follows that $\Psi$ is trivial on $A$ by Lemma 1 of [4].

In the other case there is some $t: A \rightarrow 2$ such that there is no $f$ such that $R(t, f)$ holds. This implies that for every $q \geq\left(s^{\prime} \cup t, p^{\prime}\right)$ there are infinitely many integers in $\Psi^{*}(A)$ whose membership in $Z$ is not decided by $q$. Genericity implies that $\left(s^{\prime} \cup t, p^{\prime}\right) \Vdash_{\mathbb{S} * \mathbb{P}}$ " $Z \cap \Psi^{*}(A) \not \equiv^{*} \Phi^{*}\left(t^{-1}\{1\}\right)$ " which is a contradiction to the assumption that $(s, p) \Vdash_{\mathbb{S} * \mathbb{P}}$ " $\Psi^{*}\left(X_{G}\right)=Z$ ".

Theorem 3.1. Let $V$ be a model of $2^{\aleph_{0}}=\aleph_{1}$. If $\mathbb{P}_{\omega_{2}}$ is the countable support iteration of partial orders as in Lemma 3.1 such that $\mathbb{P}_{\alpha+1}=\mathbb{P}_{\alpha} * \mathbb{S}$ for a stationary set of $\alpha \in \omega_{2}$, then $V[G]$ is a model where every automorphism of $\mathcal{P}(\mathbb{N}) /[\mathbb{N}]<\aleph_{0}$ is somewhere trivial for every $G \subseteq \mathbb{P}_{\omega_{2}}$ which is generic over $V$. (Hence, it is consistent that every automorphism of $\mathcal{P}(\mathbb{N}) /[\mathbb{N}]^{<\aleph_{0}}$ is somewhere trivial and $\mathfrak{d}=$ $\aleph_{1}$.)

Proof. Any automorphism can be reflected on a closed unbounded subset of $\omega_{2}$ consisting of ordinals of uncountable cofinality. If $\alpha$ is any ordinal such that $\mathbb{P}_{\alpha+1}=$ $\mathbb{P}_{\alpha} * \mathbb{S}$, then Lemma 3.2 can be applied to show that the automorphism can not be extended any further.

Finally, it should be remarked that the hypothesis of Lemma 3.1 can be extended to include various other partial orders. However, in light of the lack of immediate applications and the technical difficulties required to establish this, the proof will be provided elsewhere.

\section{REFERENCES}

[1] Tomek Bartoszyński and Haim Judah. Set Theory - On the structure of the real line. A K Peters, 1995. MR 96k:03002

[2] S. Shelah and J. Steprāns. Non-trivial homeomorphisms of $\beta N \backslash N$ without the Continuum Hypothesis. Fund. Math., 132:135-141, 1989. MR 90h:54015 
[3] S. Shelah and J. Steprāns. Somewhere trivial autohomeomorphisms. J. London Math. Soc. (2), 49:569-580, 1994. MR 95f:54008

[4] B. Velickovic. Definable automorphisms of $\mathcal{P}(\omega) /$ fin. Proc. Amer. Math. Soc., 96:130-135, 1986. MR 87 m:03070

[5] Boban Veličković. OCA and automorphisms of $\mathcal{P}(\omega) /$ fin. Topology Appl., 49(1):1-13, 1993. MR 94a:03080

Department of Mathematics, Rutgers University, Hill Center, Piscataway, New JerSEY

Current address: Institute of Mathematics, Hebrew University, Givat Ram, Jerusalem 91904, Israel

E-mail address: shelah@math.rutgers.edu

Department of Mathematics, York University, 4700 Keele Street, Toronto, Ontario, CANADA M3J 1P3

E-mail address: steprans@yorku.ca 